\title{
Devices for Photodynamic Studies Based on Light-Emitting Diodes
}

\author{
Gennady A. Meerovich ${ }^{1,2^{*}}$, Kirill G. Linkov ${ }^{1}$, Alexei V. Nekhoroshev ${ }^{2}$, Alexander V. Borodkin ${ }^{1}$, \\ Ekaterina V. Akhlyustina2, Ivan P. Angelov ${ }^{3,4}$, Denis A. Glechik ${ }^{2}$, and Victor B. Loschenov ${ }^{1,2}$ \\ ${ }^{1}$ Prokhorov General Physics Institute of the Russian Academy of Sciences, 38 Vavilov str., Moscow 119991, Russia \\ ${ }^{2}$ National Research Nuclear University "MEPHI", 31 Kashirskoe shosse, Moscow 115409, Russia \\ ${ }^{3}$ Institute of Organic Chemistry with Centre of Phytochemistry, Bulgarian Academy of Sciences, \\ 9 acad. Georgi Bonchev str., Sofia 1113, Bulgaria \\ ${ }^{4}$ Institute of Electronics, Bulgarian Academy of Sciences, 72 Boulevard Tzarigradsko shose, Sofia 1784, Bulgaria \\ * e-mail: gennadymeerovich@gmail.com
}

\begin{abstract}
Light sources based on light-emitting diodes (LED) can be created for almost any wavelength suitable for exciting photosensitizers (PS). This paper presents the main approaches to develop LED-based light sources for wellcontrolled photodynamic exposure and the results of their implementation. High power density LED light sources were designed and tested for both in vitro and in vivo and photodynamic studies, as well as for irradiation of various pathological foci during photodynamic therapy. (C) 2021 Journal of Biomedical Photonics \& Engineering.
\end{abstract}

Keywords: light emitting diode; power density; wavelength; light divergence; distribution of light energy.

Paper \#3457 received 8 Sep 2021; revised manuscript received 25 Oct 2021; accepted for publication 26 Oct 2021; published online 11 Nov 2021. doi: 10.18287/JBPE21.07.040308.

\section{Introduction}

The photodynamic action (PDA) causes photo-oxidative destruction of molecular structures important for functioning of tumor cells and pathogens such as bacteria and viruses. Photosensitizer (PS) molecules catalyze the generation of reactive oxygen species (ROS) upon excitation with light. Light transfers the energy necessary for this process to the medium containing the PS. However, only part of the light energy is absorbed by PS. The relative absorbed photodynamic dose (RAPD) [1] depends on properties of PS (extinction, concentration in a sensitized medium, wavelength and shape of the spectral contour at this concentration) and properties of the medium (scattering and absorption by endogenous pigments and other components, in particular, hemoglobin and water). PDA is enhanced with increasing the volume density of the energy absorbed by PS, which depends on the light intensity and the RAPD.

Light-emitting diodes (LED) are widely used as light sources for PDA [2-7]. These sources provide light with high intensity, and their light spectrum is narrow $\left(\Delta \lambda_{\text {LED }}=20-30 \mathrm{~nm}\right)$ in comparison with the spectral width of the PS absorption band. LED can generally be created with most wavelengths suitable for PS excitation (at least in the red and near infrared spectral ranges).
Modern LEDs form a diverging beam of light with a power of up to hundreds of milliwatts coming out of the surface of chip with dimensions of less than $2 \mathrm{~mm}^{2}$. The light power density is uniformly distributed over the chip surface, but non-uniformly distributed over the angle. Installing a short-focus semi-spherical lens close to the chip can significantly reduce the angle in which the main part of the light power is concentrated, but an angle distribution of power density after this lens remains nonuniform. Typically, the such LED with a lens has a bellshaped angular distribution of power, close to a Gaussian distribution, and consequently the light power density distribution of such a single high power LED on irradiated surface of any significant size will be nonuniform. Angular divergence of LED light can be characterized by the value of the characteristic angle at which the light intensity decreases to the level of $1 / \mathrm{e}$ of the value perpendicular to the LED surface.

For many applications, it is important to ensure a uniform light power density over the irradiated area (in particular, for multi-sample PDA studies in microplates, it is advantageous to be able to uniformly irradiate a large area with dimensions of at least $8 \times 12 \mathrm{~cm}$ or $14.5 \mathrm{~cm}$ in diameter). This requires designing special LED sources with the proper spatial uniformity of the light power density at some distance. As a solution of this problem a 
matrix device was created that contained a large number of identical LEDs [8], however, it was an insufficient approach since power density in the peripheral part of irradiated zone at about half of the power density at the center of it. To compensate for this inhomogeneity, it was necessary to use separate regulation and power supply of LEDs [9], which significantly increased the cost and complicated the devices. But such a device, being a set of local LEDs, can align values only in those specific wells, the centers of which are located strictly along the axis of the LED light beam. Due to the finite geometric dimensions of the LED, it is almost impossible to equalize the power density in all wells of multi-well (96 or more wells) plates.

The main approaches to the use of LED for PDA are related to the spatial distribution of the exciting light energy, which should ensure its efficient use for PS excitation. The sources of light based on LED can be used to irradiate tumor foci of both small (about $1 \mathrm{~cm}$ ) sizes and superficial foci of a large area. An important task in photodynamic therapy of tumors is to achieve a high intensity of PDA in the deep layers of tumor nodes, which makes it necessary to provide high values of the light energy absorbed by the PS in these layers. The main loss of light energy in biological tissue is caused by the absorption of endogenous substances (hemoglobin and water), the total absorption of which is minimal in the range of 660-950 nm (so-called "spectral window of biotissue transparency"). The absorption of melanin (in the skin of dark-skinned patients, as well as in PDT of melanoma) can also decrease the density of light power in the deep layers of the tumor and reduce the effectiveness of PDT. Another approach of using LEDs for PDA is photodynamic inactivation (PDI) of pathogens $[10,11]$. PDI is usually carried out on surfaces of a sufficiently large area in thin layers of a sensitized biological medium. PDA in thin sensitized layers is also executed during in vitro screening studies of PS, that are carried out to assess the sensitivity of different cells to PDT, to study its mechanisms and properties of PS $[6,12]$. For small layer thickness and low PS concentrations (less than $10 \mu \mathrm{M}$ ) used for such a PDA, a significant part of the light can pass through the layer without participating in the PDA process. This loss results in decreased PDA efficiency. It can also lead to errors in screening studies of PS with various extinction and concentration since both the losses and the amount of the absorbed energy in the sensitized layer for various PS will be different. To increase the efficiency of the PDA in thin layers, the LED wavelength should be close to the spectral absorption maximum of the PS [13].

\section{Results and Discussion: New LED Devices for PDA}

Our study used a number of PS with excitation maxima within ranges of 662-665 nm (methylene blue, as well as chlorin derivatives Radachlorin, Photoran and Photolon), 670-675 nm (Photosens), 682-686 nm (Cholosens), 760-765 nm (derivatives of synthetic bacteriochlorin).
The wavelength of excitation light was usually chosen near the spectral maximum of the PS absorption band. Light sources of suitable wavelengths required for PS excitation were designed using LED models (LC-1DR-G30 for the spectral range of $660-665 \mathrm{~nm}$, LC-1IR1-G42 for 680-690 nm, LC-1IR3-G42 for 760-770 nm) manufactured by Shenzhen Cai Mingzhu Co., LTD (China).

Power and power density of the incident light as well as homogeneity of its distribution within the illuminated area were evaluated with the PM180T Power Meter (Thorlabs GmbH, Germany). Spectra of emission of illumination devices were recorded using fiber optic spectra-analyzer LESA-01-BIOSPEC (Biospec Ltd, Russia).

A range of compact devices intended for different spectral ranges based on single powerful LED with an optical projecting system the chip image onto the irradiated foci have and a miniature cooling system has been created for the irradiation of small tumor foci. Developed devices (Fig. 1) provided irradiation of areas with dimensions that can be adjusted by the optical system from $0.8 \times 0.8 \mathrm{~cm}$ to $3 \times 3 \mathrm{~cm}$. The light power of devices reached $160 \mathrm{~mW}$; the inhomogeneity of the power density over the irradiated area in all cases did not exceed $\pm 7 \%$. Optimum light power density and uniformity was achieved at a distance of $8-12 \mathrm{~cm}$ from the device. Such a compact powerful device is very useful and convenient both in clinical application for photodynamic therapy of small skin, head and neck tumors, as well as and in experimental oncology for PDT of model tumors of laboratory animals.

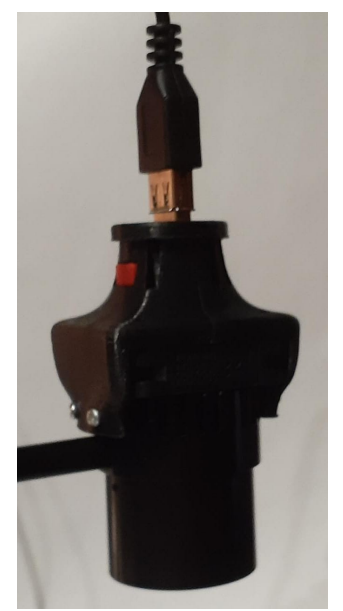

Fig. 1 Device based on single powerful LED.

A powerful device based on a group of LED, equipped with a heat sink in the shape of a segment of a spherical surface, has been developed to irradiate large surface tumor nodes. The optical axes of the LED light beams with a light divergence of about $48^{\circ}$ are directed to the center of the spherical surface. This design made it possible to evenly distribute the light of 36 powerful LED in the irradiated area while keeping compact arrangement of LEDs and providing good conditions for heat 

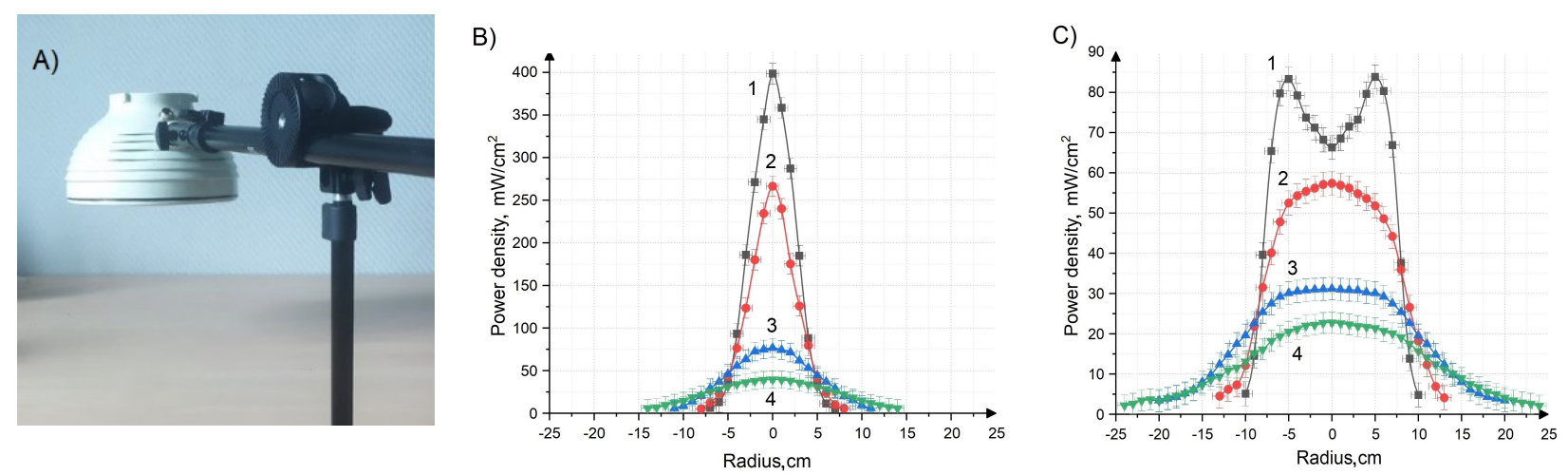

Fig. 2 Powerful device based on a group of LED: A) device with a manipulator for adjusting the aiming at the irradiated area; B) bell-shaped distribution of power density; C) uniform distribution of power density. Distances between the device and irradiated surface asfollows: (1) $8 \mathrm{~cm}$, (2) $12.5 \mathrm{~cm}$, (3) $18.5 \mathrm{~cm}$, (4) $22 \mathrm{~cm}$.

dissipation (Fig. 2A). The device forms a light spot in the irradiation zone with an area of about $20 \mathrm{~cm}^{2}$ and a bellshaped distribution of the power density reaching about $400 \mathrm{~mW} / \mathrm{cm}^{2}$ at the center of the spot (Fig. 2B). Such a powerful device is used for photodynamic therapy of large superficial tumors, in particular, basal cell skin cancer.

The above device was modified for highly uniform irradiation of large areas, in particular, for in vitro multisample PDA studies in standard 96-well or similarly sized microplates. To increase the uniformity of the power density on the irradiated plate, the LEDs located near the center of the in the mount had lower divergence of light beam of about $23^{\circ}$ ), while LEDs at the periphery-larger of about $48^{\circ}$. Such arrangement provided a high uniformity of a power density of light with an average value of about $25 \mathrm{~mW} / \mathrm{cm}^{2}$ and deviation below to $\pm 10 \%$ over an area exceeding $100 \mathrm{~cm}^{2}$ (Fig. 2C).

Increasing this distance will make it possible to implement uniform irradiation of a sufficiently large area (about $1000 \mathrm{~cm}^{2}$ ) with light power density of about $2.5 \mathrm{~mW} / \mathrm{cm}^{2}$, which can be used for photodynamic antiviral disinfection [11].

Another group of light sources has been developed based on single high-power LEDs of different spectral ranges to use in studies of PDA in vitro in individual optical cells of $1 \times 1 \mathrm{~cm}$ size. The optical system of the device projected the image of the chip onto the surface of the sensitized layer in the optical cell containing sensitized biological objects and provided uniform PDA in them. The devices are equipped with a system of optical fibers (Fig. 3), which also made it possible to investigate the fluorescence, absorption and scattering in sensitized layer during PDA, as well as to evaluate the concentration and state of irradiated PS and biological micro-objects. This has been used to study the photodynamic inactivation of viruses and bacteria $[11,14]$.

Developed devices were powered from adapters through programmable timers to ensure a given dose of light irradiation, which can also operate in a periodic mode to study the dependence of the effect on the irradiation dose and for group studies.
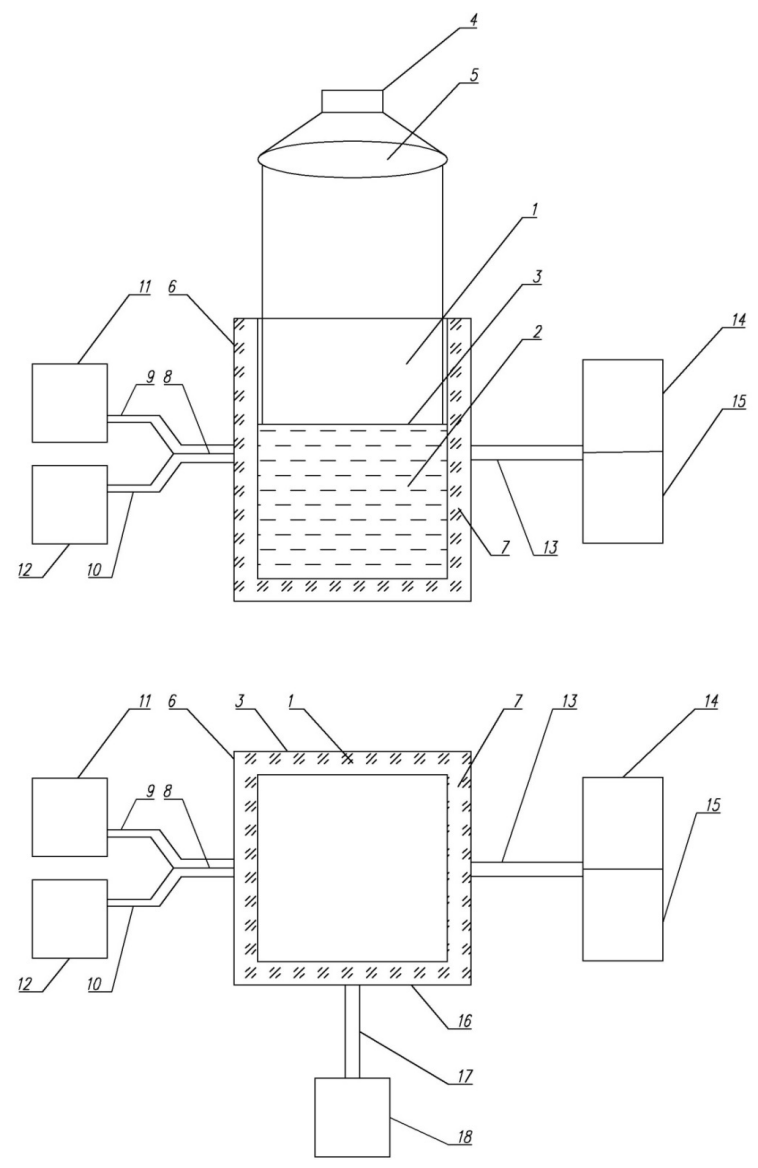

Fig. 3 Functional diagram of a device for in vitro PDA research: 1 - optical cell; 2 - sensitized layer containing biological micro-objects; 3 - surface of layer; 4 - LED; 5 -optical projecting system; $6,7,16$ - transparent walls of optical cell; 8 - optical fiber bundle; 9 - optical fiber connected to the laser 11 output; 10 - fiber connected to the input of the spectra-analyzer; 11-laser for fluorescence excitation; 12 - spectra-analyzer LESA-01BIOSPEC; 13 - optical fiber connected to the output of a broadband light source (for absorption studying) or to the output of an additional laser (for scattering studying); 14 - broadband light source; 15 -laser for scattering studying; 17 - optical fiber connected to the input of the dynamic light scattering spectrometer 18 . 


\section{Conclusion}

A range of LED sources have been developed with high light power and power density for excitation of photosensitizers of different spectral ranges. They can be widely used as light sources for in vivo and in vitro photodynamic research and various PDT applications.

\section{Disclosures}

All authors declare that there is no conflict of interests in this paper.

\section{Acknowledgments}

The research of E. Akhlyustina was funded by RFBR according to the project \#20-04-60084.

The research of G. Meerovich and I. Angelov was funded by RFBR and BNSF according to the bilateral project (RFBR \#20-52-18008, BNSF \#KP- 06- Russia/9- 2020).

\section{References}

1. B. W. Pogue, L. Lilge, M. S. Patterson, B. C. Wilson, and T. Hasan, "Absorbed photodynamic dose from pulsed versus continuous wave light examined with tissue-simulating dosimeters," Applied Optics 36(28), 7257-7269 (1997).

2. V. B. Loschenov, K. G. Linkov, T. A. Savelieva, M. V. Loschenov, S. S. Model, and A. V. Borodkin, "Hardware and tool equipment for fluorescence diagnostics and photodynamic therapy," Photodynamic Therapy and Photodyagnosis 2(3), 17-25 (2013) [in Russian].

3. J. Neupane, S. Ghimire, S. Shakya, L. Chaudhary, and V. P. Shrivastava, "Effect of light emitting diodes in the photodynamic therapy of rheumatoid arthritis," Photodiagnosis and Photodynamic Therapy 7(1), 44-49 (2010).

4. A. Erkiert-Polguj, A. Halbina, I. Polak-Pacholczyk, and H. Rotsztejn, "Light-emitting diodes in photodynamic therapy in non-melanoma skin cancers - own observations and literature review," Journal of Cosmetic and Laser Therapy 18(2), 105-110 (2016).

5. E. Sorbellini, M Rucco, and F. Rinaldi, "Photodynamic and photobiological effects of light-emitting diode (LED) therapy in dermatological disease: an update," Lasers in Medical Science 33(7), 1431-1439 (2018).

6. R. I. Yakubovskaya, E. A. Plotnikova, A. D. Plyutinskaya, N. B. Morozova, V. I. Chissov,E. A. Makarova, S. V. Dudkin, E. A. Lukyanets, and G. N. Vorozhtsov, "Photophysical properties and in vitro and in vivo photoinduced antitumor activity of cationic salts of meso-tetrakis (N-alkyl-3-pyridyl)bacteriochlorins," Journal of Photochemistry and Photobiology B: Biology 130, 109-114 (2014).

7. I. Meerovich, M. G. Nichols, and A. K. Dash, "Low-intensity light-induced paclitaxel release from lipid-based nanodelivery systems," Journal of Drug Targeting 27(9), 971-983 (2019).

8. A. V. Belikov, Yu. V. Semyashkina, M. A. Modin, and D. R. Zhubrev, "LED illuminator for photodynamic therapy of onychomycosis," Scientific and Technical Bulletin of Information Technologies, Mechanics and Optics 17(5), 782-789 (2017).

9. N. Yu. Shilyagina, I. V. Balalaeva, I. V. Turchin, N. M. Shakhova, V. I. Plekhanov, and A. A. Brilkina, A device for studying the light activity of photosensitizers in vitro, Utility model No. RU 150108, 27.01.2015.

10. M. G. Strakhovskaya, G. A.Meerovich, A. N. Kuskov, S. A. Gonchukov, and V. B. Loschenov, "Photoinactivation of coronaviruses: going along the optical spectrum," Laser Physics Letters 17(9), 093001 (2020).

11. K. Sharshov, M. Solomatina, O. Kurskaya, I. Kovalenko, E. Kholina, V. Fedorov, G. Meerovich, A. Rubin, and M. Strakhovskaya, "The Photosensitizer Octakis(cholinyl)zinc Phthalocyanine with Ability to Bind to a Model Spike Protein Leads to a Loss of SARS-CoV-2 Infectivity In Vitro When Exposed to Far-Red LED," Viruses 13(4), 643 (2021).

12. G. A. Meerovich, E. V. Akhlyustina, I. G. Tiganova, E. A. Lukyanets, E. A. Makarova, E. R. Tolordava, O. A. Yuzhakova, I. D. Romanishkin, N. I. Philipova, Yu. S. Zhizhimova, Yu. M. Romanova, V. B. Loschenov, and A. L. Gintsburg, "Novel Polycationic Photosensitizers for Antibacterial Photodynamic Therapy," in Advances in Experimental Medicine and Biology, Springer, New York, 1-19 (2019).

13. G. Meerovich, I. Romanishkin, E. Akhlyustina, M. Strakhovskaya, E. Kogan, I. Angelov, V. Loschenov, and E. Borisova, "Photodynamic Action in Thin Sensitized Layers: Estimating the Utilization of Light Energy," Journal of Biomedical Photonics \& Engineering 7(4), 040301 (2021).

14. G. A. Meerovich, E. V. Akhlyustina, D. S. Denisov, E. G. Kholina, E. A. Makarova, E. A. Lukyanets, V. B. Loschenov, and M. G. Strakhovskaya, "Photodynamic inactivation of Escherichia coli bacteria by cationic photosensitizers," Laser Physics Letters 18(11), 115601 (2021). 